\title{
DE LA CRISIS AL DESAFÍO DE REINVENTAR LA EDUCACIÓN SECUNDARIA
}

\author{
Silvia Andrea Vázquez (Universidad Nacional de Luján- \\ Confederación de Trabajadores de la Educación de la República Argentina)* \\ savazquez@fibertel.com.ar
}

Recibido: 22/07/2011 Aceptado: 26/10/2011

\section{Resumen}

Este trabajo presenta un conjunto de ideas que apuntan a enriquecer el debate respecto de la necesaria transformación de la educación secundaria en nuestro país.

El contenido de las propuestas se nutre de conocimientos que se desarrollan en la propia praxis de la Confederación de Trabajadores de la Educación de la República Argentina (CTERA), enriquecido por el aporte de un trabajo de investigación realizado entre dicha organización sindical y la Universidad Nacional de Luján.

En la investigación se sistematizaron cuatro experiencias educativas de nivel secundario: una Escuela de Reingreso de la Capital Federal, un Centro Educativo para Adultos de Río Negro, un Bachillerato Libre Intercultural de Chaco, y una Escuela Secundaria Básica de Buenos Aires. La reflexión sobre prácticas y discursos de cada institución permitió identificar rupturas y continuidades en relación con rasgos que hemos caracterizado como tendencias hegemónicas del nivel secundario.

A partir de este primer análisis se sugieren líneas de acción sobre aspectos necesarios para pensar políticas públicas que reconfiguren la escuela secundaria: reconfigurar la identidad pedagógica de la educación secundaria, hacer de la inclusión una ideología institucional, refundar las bases de la autoridad de los docentes, revisitar las prácticas de la enseñanza y generar condiciones para el trabajo docente colectivo.

\section{Palabras claves}

Educación Secundaria - Políticas Educativas - Inclusión - Autoridad Pedagógica - Organización Escolar.

* Licenciada en Ciencias de la Educación. Facultad de Filosofía y Letras. Universidad Nacional de Buenos Aires. Investigadora del Instituto de Investigaciones Pedagógicas de CTERA y Directora de la Colección Formación y Trabajo Docente. Ediciones CTERA. 


\section{Abstract}

This paper presents a set of ideasthey aim to enrich the debate on the necessary transformation of the school in our country.

The content of the proposals draws on knowledge developedown praxis of the Confederation of education workers of the Argentina Republic (CTERA), enriched by the contributiona research project carried out between the trade union organization and the National University of Luján.

Research is systematized four experienceseducational secondary level: a school of re-entry of the Federal Capital, an educational center for adults of Rio Negro, a free Intercultural baccalaureate of Chaco, and a basic secondary school of Buenos Aires.Reflection on practices and discourses of each institution identified ruptures and continuities in relation to traits that we have characterized as hegemonic level trends secondary.

From this first analysis isthey suggestlines of action on aspects necessary to think about public policies that reconfigured the secondary school: reconfigure the pedagogic identity of secondary education, make an institutional ideology of inclusion, refound the foundations of the authority of teachers, revisiting the teaching practices and create conditions for teaching work collective.

\section{Key words}

Secondary Education - Education Policy - Inclusion - Pedagogical Authority School Organization.

\section{Introducción}

Las ideas sobre la educación secundaria que dan lugar a este trabajo surgen de un proceso de construcción colectiva de conocimientos desarrollado en los ámbitos de producción político-pedagógica de Confederación de Trabajadores de la Educación de la República Argentina (CTERA), enriquecido por el aporte de un proyecto de investigación que, bajo mi dirección, se realiza de manera conjunta con la Universidad Nacional de Luján (1).

Esta conjunción de esfuerzos por construir una lectura crítica de la realidad entre un ámbito académico y otro gremial logra generar un diálogo de saberes de notable potencia política (como se explicitará más adelante).

Estas reflexiones se sitúan en un particular momento de la política educativa nacional, en el cual la escuela secundaria parece estar en el centro de las preocupaciones y redefiniciones. Para superar los diagnósticos acerca de la "crisis" de este nivel del sistema, hoy se hace necesario producir un profundo cambio cultural, que fracture los sentidos comunes respecto del valor social de la educación secundaria, y anticipe formas y contenidos de una escolaridad diferente. 


\section{El conocimiento y la organización sindical}

Comenzaremos describiendo brevemente un rasgo, poco usual en el ámbito gremial, y que distingue a la CTERA: la particular vinculación entre la producción de conocimientos y el accionar político sindical. Asunto que permite comprender el aporte de esta organización a la construcción de los contenidos de una política educativa, uno de los procesos que nos interesa describir en este trabajo.

Esta confederación de sindicatos docentes se ha caracterizado por concebir la lucha política como la articulación de la pelea reivindicativa sectorial con la disputa ideológica por la dirección y el sentido de las políticas educativas. En su modelo de construcción sindical la producción de un conocimiento desde los propios trabajadores y la acción política van juntos.

Durante los noventa, fue visible la capacidad de CTERA de aglutinar las resistencias docentes y de otros sectores sociales a las políticas educativas neoliberales - representadas en nuestro país por las leyes de Transferencia Educativa, los Pactos Federales y la Ley Federal de Educación - que intentaron desmembrar el sistema de educación pública a través del ajuste y el desfinanciamiento. Las acciones políticas y los conocimientos construidos en esa resistencia contribuyeron a crear condiciones materiales y simbólicas para derogar de la LFE, e incidir en la construcción de nuevas bases legales, imprescindibles para el rediseño de las políticas públicas que deben sustentar el derecho social a la educación: ley de Financiamiento Educativo, ley Nacional de Protección Integral de los Derechos de los Niños, Niñas y Adolescentes (26 061/05), Ley de Educación Nacional (26.206/06) y la puesta en vigencia de leyes o ámbitos formales de Negociación Colectiva de Trabajadores Docentes.

El amplio debate democrático que precedió la formulación de la LEN logró que la misma contuviera dos de las principales reivindicaciones populares respecto de la educación secundaria.

- la extensión de la obligatoriedad a la totalidad del nivel, re-situando al Estado en el lugar de garante del derecho social, a cargo de políticas educativas y socioeducativas que posibiliten el acceso, permanencia y egreso de todos los adolescentes y jóvenes;

- y la recuperación de la unidad pedagógica de una educación secundaria fragmentada y anarquizada por las reformas neoliberales, como punto de partida para la redefinición del proyecto político-educativo de una escuela para todos los adolescentes y jóvenes.

Si bien el marco que regula hoy el sistema educativo nacional avanza en dirección a la ampliación de derechos, sabemos que una ley es, en cualquier caso, una condición necesaria pero no suficiente para generar transformaciones. Su eficacia instrumental y simbólica (Santos; 2003) dependerá de la fuerza social que se logre organizar, para impulsar políticas públicas que hagan efectivos los derechos conquistados, para luego concretarse en prácticas escolares. 
En caso contrario, no sería modificada la realidad escolar de los '90.

\section{Investigar el conocimiento que se produce en la práctica}

Desde la investigación nos propusimos identificar elementos que den cuenta de procesos de educación popular en cuatro instituciones de educación secundaria elegidas por mostrar a priori algunos rasgos que las distinguen de las escuelas tradicionales. Partimos del supuesto que el nivel medio, por su origen e historia, presenta como rasgos hegemónicos tendencias la fragmentación, la selectividad y el autoritarismo y buscamos en las experiencias la presencia de elementos que se alejaran o directamente revirtieran esos "mandatos" históricos, así como de otro más renuentes al cambio.

En nuestro marco conceptual explicitamos dos convicciones: que las experiencias educativas jamás son monolíticas y que las prácticas sociales y de la conciencia de los sujetos están atravesadas de múltiples contradicciones. Con estos presupuestos fuimos procesando y e interpretando el material recogido en el trabajo de campo; intentamos discernir en cada una de estas experiencias de qué manera se da la convivencia entre aspectos que se alejan de los rasgos hegemónicos del nivel medio, y aspectos en los que las intenciones transformadoras encuentran más resistencias o dificultades para concretarse (debido quizás a que los condicionamientos que constriñen las prácticas son más fuertes o la inercia de la tradición de la institución es más poderosa).

El proceso de construir categorías de análisis, sistematizar e interpretar los datos tuvo espacios compartidos con directivos y profesores de las mencionadas instituciones, intentamos que los docentes que sostienen las experiencias pudieran apropiarse de los conocimientos construidos por ellos mismos en sus prácticas, superando el rol de meros informantes o emisores de discursos, en el que tradicionalmente los pone la investigación académica.

Si bien el análisis final de los datos aún se está realizando, los indicios encontrados muestran que las rupturas que alteran la escolaridad secundaria tradicional aparecen con cierta fortaleza cuando: a) afectan tanto la materialidad de las instituciones como su universo de significaciones; b) transforman simultánea y coherentemente dimensiones como: el currículo, la organización escolar y el proceso de trabajo docente.

En un plano más concreto las experiencias analizadas presentan - aunque de manera no homogénea, ni exentas de contradicciones - transformaciones significativas en algunas dimensiones institucionales que desafían los rasgos hegemónicos de la escolaridad secundaria. Algunas de estas rupturas son:

- Poner el reconocimiento de los estudiantes en tanto sujetos históricosociales en centro de la definición del proyecto educativo institucional. Hacer que el respeto por sus derechos, sus condiciones de vida y sus capacidades/posibilidades se materialice en prácticas pedagógicas y estrategias institucionales. 
- Generar condiciones materiales y simbólicas que hagan posible una escolaridad inclusiva con el horizonte de hacer efectivo el derecho social a la educación. Los cambios en la organización escolar y las políticas socioeducativas se revelan como base material de la inclusión.

- $\quad$ Construir nuevas formas de ser docente y estudiante, democratizando las relaciones político-pedagógicas sin equiparar funciones.

- Reconocer que la producción de conocimiento pedagógico - de la planificación, implementación y evaluación de la enseñanza - es parte del proceso de trabajo docente y requiere de tiempos institucionales de trabajo colectivo.

- $\quad$ Búsqueda de mayor implicación de docentes y estudiantes con un conocimiento que construya una lectura no hegemónica de la realidad y forme sujetos de derecho/ciudadanos plenos.

\section{Conocimientos y acción política}

Desde nuestra perspectiva ideológica consideramos que el conocimiento producido en ámbitos académicos o fuera de ellos, cobra sentido en tanto pueda transponerse en propuestas de políticas públicas.

Creo asimismo que el proceso de reorientación de las políticas educativas iniciado en nuestro país en los últimos cinco años interpela y desafía nuestras trayectorias y posiciones. En tal sentido considero que para transitar el camino que va de los enunciados normativos a las políticas públicas, y de estas a las prácticas educativas que las concreten, se hace necesario un profundo cambio en los sentidos comunes y en las prácticas que producen las escuelas.

Para el caso específico de la educación secundaria habrá que generar desde el inicio, rupturas que anticipen la construcción de formas y contenidos de una escolaridad diferente. A continuación presentamos para el debate algunas de líneas de acción que podrían considerarse marcas refundacionales de otra escuela secundaria:

1. Hacer de la inclusión una ideología institucional sustentada en políticas públicas que garanticen sus condiciones de producción.

2. Reconfigurar la identidad pedagógica de la educación secundaria como un espacio-tiempo de formación de los adolescentes en tanto sujetos de la historia, la cultura y las transformaciones sociales.

3. Refundar la autoridad de los docentes, tradicionalmente sustentada por jerarquías formales o "experticia técnica", sobre la base de relaciones pedagógicas de respeto.

4. Redefinir los modos de selección y organización curricular en función de multiplicar las oportunidades de producir conocimientos como alternativa al "fracaso escolar". 
5. Generar otra organización del trabajo docente que permita reflexionar crítica y colectivamente sobre las prácticas y las representaciones escolarizadas.

\section{De la inclusión como acceso al derecho social a la educación}

La idea de inclusión resulta extraña a las concepciones con las que se definió tradicionalmente la forma y el contenido de la escolaridad secundaria. Surgida como enseñanza preparatoria para los estudios universitarios, se convirtió en el nivel que seleccionaba a "los mejores" para ocupar los puestos de mando en la sociedad, confirmando un destino socialmente predeterminado. El carácter selectivo resultó así, el "mandato" estructurante del sentido políticopedagógico del nivel.

A lo largo de casi un siglo y medio se fue ampliando el acceso a la educación secundaria de las clases medias y trabajadoras. Como explicita el estudio llevado a cabo por el Instituto de Investigaciones Pedagógicas de CTERA:

Ya indicamos que la escuela secundaria fue, desde su organización a mediados del siglo XIX y durante muchas décadas, un nivel educativo al que sólo una minoría tenía acceso, al contrario que lo que ocurría con la escuela primaria.(2) Esa característica se mantuvo hasta que, a partir de los grandes cambios sociales, económicos, políticos y culturales que transformaron a la sociedad argentina entre 1930 y 1945, con el peronismo comenzó un proceso de expansión del ingreso a la escuela secundaria. A partir de este momento se da un proceso de sostenido crecimiento de la asistencia a la escuela de los adolescentes y los jóvenes. Si en 1960 concurrían un 41,6 \% de entre 13 y 18 años,(3) para 1980 el porcentaje hacía crecido al $58 \%$, siendo en el 2001 del $81 \%$.(4) Particularmente se expandió la población que concurría a la escuela secundaria. En 1960 eran un $23,5 \%$ de los adolescentes y jóvenes. Para 1980 lo hacían el $40 \%$. Y en la actualidad el $64 \%$. El ritmo de crecimiento de nivel secundario fue mayor que el de la asistencia a la escuela en general.(5) Considerada en términos porcentuales, la cantidad de alumnos de la escuela secundaria se triplicó en los últimos cuarenta años. Pero en términos absolutos, se quintuplicó, pues los adolescentes y jóvenes que estudiaban en la escuela secundaria en 1960 eran 481.000, y en el 2001 cerca de 2.500.000." (Balduzzi. 2006. pp 12 y 13).

La masificación - que incorporó a las clases medias y trabajadoras a la educación pública - generó cambios en la forma de pensar y transitar la escuela secundaria: la imposición de contenidos hegemónicos se tensionó a partir del derecho al conocimiento instalado en la cultura popular y la aceptación rígida de la segmentación social se trastocó en expectativas de movilidad. Este proceso 
puso en evidencia que el acceso al nivel medio se había democratizado, pero al mantener la matriz selectiva del modelo escolar, los sectores populares vieron dificultadas sus posibilidades de permanecer y egresar.

La dictadura de 1976 abrió paso a las políticas neoliberales, consolidadas a través de la transferencia de escuelas secundarias y la Ley Federal de Educación; la llamada reforma educativa de los 90 fracturó y anarquizó el trayecto de la educación secundaria, imprimiendo sobre la legitimación de las desigualdades sociales el sello de la exclusión socio-educativa. El tradicional "nivel medio" de nuestro sistema educativo, luego de aplicada la reforma impulsada por la Ley Federal de Educación, quedó subdividido en segmentos con diferentes nombres y formas de implementación según las jurisdicciones provinciales: la EGB 3, escuela secundaria básica, escuela intermedia, polimodal, entre otras.

Investigaciones recientes señalan que en los últimos años este nivel se ha visto poblado de adolescentes y jóvenes provenientes de hogares de clases trabajadoras empobrecidas o directamente pobres, quienes por sus condiciones de vida estarían tradicionalmente fuera de la escuela secundaria. Sin embargo la ampliación de esta cobertura no implicó necesariamente la presencia de un proceso educativo inclusivo, ya que se hizo a expensas de la "primarización" del formato escolar del primer tramo de la secundaria - la EGB3 -, al cabo del cual estos estudiantes reproducen las historias de "fracaso" o "abandono".

Según algunas interpretaciones académicas, los estudiantes provenientes de los sectores sociales mencionados tienen, además de serias restricciones económico-sociales para concluir la secundaria, dificultades vinculadas con los "arbitrarios culturales" que circulan en las escuelas para incorporar el "capital cultural" que pueden obtener en dicho nivel. Por otro lado variados testimonios de los docentes manifiestan percepciones similares, en tanto consideran que buena parte del aumento de la complejidad y conflictividad de sus escuelas está vinculado con la falta de respuestas adecuadas a estos "nuevos" estudiantes.

Al asumir un posicionamiento crítico se advierte la inconsistencia de una política educativa que promete demagógicamente un lugar a todos dentro de las escuelas sin modificar el carácter segregativo inscripto en diversas lógicas y dinámicas institucionales, en la tradición selectiva del currículo y en la concepción meritocrática presente en los mecanismos internos de evaluación y promoción. Y fundamentalmente sin el aumento de la inversión y el acompañamiento de políticas socioeducativas que garanticen el acceso y egreso, y sostengan la permanencia de los estudiantes de sectores populares.

Estas consideraciones permiten afirmar que la inclusión es algo más que aumentar la matrícula y los porcentajes de permanencia, o disminución de situaciones de abandono. Debe ser considerada el primer peldaño en la posibilidad de democratizar el acceso y sostener la escolaridad de aquellas clases y sectores sociales tradicionalmente despojados de sus derechos. 
Desde la perspectiva del derecho social, es el Estado el garante y responsable de implementar y sostener las políticas públicas que garanticen las condiciones para la efectiva extensión de la escolaridad obligatoria hasta el nivel secundario. Entendemos esta obligatoriedad contemplada en la nueva Ley de Educación Nacional como el piso a partir del cual debe discutirse una escuela secundaria distinta.

Construir alternativas pedagógicas a la exclusión implica cambios estructurales en el sentido, el contenido y las formas de la educación secundaria; requiere un proceso de transformación cultural que debe estar sustentado en condiciones materiales y simbólicas de producción. Implica simultáneamente incluir y formar subjetividades críticas. Es decir, hacer de la inclusión una ideología institucional que forme a un sujeto capaz de comprender y pelear por el conjunto de los derechos sociales y humanos, y no sólo por "su" derecho a la educación.

En tal sentido considero que el formato escolar no puede ser visto, por sí solo, como el responsable de sostener la inclusión sino que su potencialidad radica en permitir el despliegue de prácticas docentes que le dan una orientación diferente al funcionamiento escolar. Cabe remarcar, entonces, la importancia de los dos componentes combinados: la materialidad del formato (con horas institucionales para algunos docentes, espacios curriculares optativos, régimen diferente de cursada y aprobación, trayectos personalizados, etc.) y la cultura institucional inclusiva como una direccionalidad ético-política instalada por docentes comprometidos que aspiran a generar una práctica distinta a la tradicional selectividad del nivel.

\section{La secundaria como proyecto cultural}

Distintas perspectivas comparten hoy la percepción de que la escuela secundaria es un ámbito escasamente interesante o amigable; un espacio-tiempo difuso e incierto, donde lo importante estuvo antes o va a estar después; donde su tránsito parece haber perdido sentido... En este contexto las conductas de muchos estudiantes adolescentes serán el emergente de un sin-sentido que cuesta formular como pregunta y que suele intentar resolverse por la negación o la sanción.

La suma de cambios culturales que atraviesan nuestra época y de políticas neoliberales han hecho entrar en crisis la eficacia de una escuela destinada a legitimar la segmentación social a través de mecanismos de segregación y expulsión, de la desigualdad jerárquica entre profesores y estudiantes y su consecuente autoridad disciplinadora, de la primacía de la formación enciclopedista justificatoria de una cultura meritocrática, entre otros. La caída de la eficacia tradicional destituye los sentidos que dieron contenido a una escuela secundaria, reduciéndola a pura forma: una organización escolar rígida y vacía, un orden 
normativista, y condiciones de enseñar y aprender cada vez más deterioradas y deteriorantes, tanto para los chicos como para los docentes.

La discusión sobre el sentido de la educación secundaria muchas veces se circunscribe a debatir si siguen teniendo vigencia los fines tradicionales - formar para seguir estudios académicos, para ejercer las obligaciones ciudadanas, para un mejor empleo que posibilita el ascenso social -, metas que adquieren significación desde "afuera" o "después" del tránsito por la educación media. Sin embargo, poco hay reflexionado respecto de qué marcas produce la experiencia misma de escolaridad en las subjetividades adolescentes que el proceso educativo ayuda a cimentar.

El discurso clásico sobre los fines clásicos de la educación secundaria parece olvidar que las metas instrumentales a ser alcanzadas sólo pueden explicar una parte del "sentido", y que no pueden obviarse los significados construidos en el proceso mismo de estar y hacer con otros, en el propio tránsito por la escuela secundaria. Sentidos de identidad, diferencia, pertenencia, ajenidad. Constitutivos y constituyentes de una forma de ser de la institución y los sujetos.

Los adolescentes de hoy deben sumar a la complejidad de su etapa existencial el hecho de que las condiciones socioculturales históricas en las que desenvuelven su vida parecen complicar dicho proceso. Sobre todo el rasgo social que, ante la caída de paradigmas, eficacias y certezas, deja vacante la posición de adulto, el lugar de la autoridad, la ley, la prohibición, sin la cual la conformación subjetiva del adolescente se hace sumamente dificultoso.

El mercado encuentra en los adolescentes de las clases medias a los consumidores natos que el sistema necesita, y los convierte en modelos sociales cuya imagen física, sus códigos, su sentido de la realidad se busca imponer al conjunto social. Por su parte, miles jóvenes pobres e indigentes, son incitados a la violencia ante la imposibilidad de cumplir el mandato social de 'consumir para ser'; al tiempo que el poder hegemónico y sus voceros construyen un discurso que homologa adolescencia pobre con delincuencia e inseguridad. Instalan, así, un mecanismo de culpabilización del adolescente -'el adolescente es peligroso' - por el cual se busca que la sociedad, en lugar de cuidar y proteger la adolescencia como "prole universal", se proteja de ella... (CTERA. 2008; pag 8).

Quizá por esto sea necesario reconfigurar la identidad pedagógica de la educación secundaria como un espacio-tiempo de formación de los adolescentes en tanto sujetos de la historia, la cultura y las transformaciones sociales. Reinventar el sentido de la escuela secundaria pensada en sí misma como un ámbito de producción cultural donde, guiados por los educadores, los estudiantes puedan protagonizar sus propios proyectos. 
Asumir el desafío de volver a pensar la escuela secundaria en tanto proyecto cultural; de valorizar el estar en la escuela y estar con otros como marca y experiencia cultural, frente a la vacuidad que pueden tener otras formas mercantilizadas de lo colectivo.

Tal vez - parafraseando las reflexiones de nuestra compañera Marta Santore-, se trate de que los educadores seamos capaces de trasmitir nuestra experiencia social a los jóvenes estudiantes, “...evitando que una peligrosa amenaza se cumpla, la de ruptura de la continuidad de la vida cultural y sus formaciones sociales cuando las generaciones precedentes se cuidan de la siguiente en lugar de protegerla..." (Santore, M; 2010, p. 3).

Se trata, en definitiva de que los estudiantes puedan formarse en "el encuentro y malentendido entre generaciones", no para reproducir nuestros horizontes o aceptar nuestras formas de estar y hacer en el mundo, sino para que a partir de conocerlas y desecharlas, puedan crear y multiplicar las propias.

\section{Refundar la autoridad de los docentes}

Así como empieza a verse la necesidad de redefinir el sentido de la educación secundaria para poder interpelar e incidir en la formación de nuevas subjetividades adolescentes, es necesario problematizar las formas en que los profesores han desplegado su autoridad como educadores.

La trama de relaciones que constituyen una institución educativa resulta del modo de ser de un orden social que condiciona lógicas y dinámicas internas, capaces de producir habitus escolares específicos. De ellos depende que se constituyan o no diversos sentidos de pertenencia, autoridad, referencia, identidad.

Entre las "eficacias" de la escuela secundaria tradicional, algunos autores ubican la capacidad de crear un sentido de pertenencia - construido sobre relaciones pedagógicas que reproducían las desigualdades sociales y operaban un proceso de "distinción"-, creando lazos identitarios comunes entre los selectos o seleccionados.

En un mundo donde las instituciones empiezan a ser fuertemente cuestionadas y entra en crisis ese sentido de pertenencia identificatorio, los adolescentes jaquean las "autoridades" escolares instituídas: sea lo que dice el libro de texto, la norma disciplinaria, o la palabra del docente.

Algunos creen ver que se pone en cuestión la matriz de las relaciones de saber-poder instauradas por le escuela moderna. Otros inhabilitan ese proceso-movimiento de cambios incipientes, augurando la restauración de las "autoridades" nostálgicas (nostalgia que borronea la rebeldía experimentada en propias adolescencias escolarizadas). Existen quienes advierten que las políticas de profundización de la desigualdad y la exclusión - que han precarizado material y simbólicamente las condiciones de vida de instituciones y 
personas -, parecen condenar a las escuelas al inmovilismo que impide tanto retrotraerse como avanzar.

Sin pertenencias ni identidades no hay posibilidad de que los sujetos que pasan por la escuela secundaria, quieran arraigarse, echar raíces, comprometerse o simplemente permanecer. Abordar la profundidad del sin-sentido y des-arraigo se hace imperioso para refundar nuevas autoridades.

Es necesario que la escuela secundaria se re-autorice a producir huellas vitales en los sujetos que la habitan, y no sólo se afane y ufane por alcanzar metas, objetivos observables, conductas mensurables, competencias negociables en el mercado global.

Es imprescindible que su tránsito produzca pertenencia, ganas de ser parte, de estar dentro. Y para eso necesita que allí pase algo vitalmente significativo tanto para estudiantes como para docentes. Que se sientan autorizados a convocar, a intervenir, a proponer o afrontar desafíos tanto desde el lugar de enseñar como de aprender.

Y en tanto institución educadora debiera dejar marcas específicas que revaloricen el conocimiento y la cultura, que redescubran la potencia política de trabajos como enseñar, pensar, experimentar, conocer, aprender.

En este proceso de verdadera reinvención de relaciones político-pedagógicas, la posición de educador y educando no pueden pensarse como unos lugares fijos, al estilo de los roles instituidos en el siglo XIX. Debemos construir colectivamente nuevos modos de ser profesor, de ser estudiante, pensados como desempeños necesariamente redefinidos a la luz de la cultura actual, pero nunca intercambiables al punto de su disolución.

Los docentes podremos recrear las bases de nuestra autoridad si sentimos que queremos y tenemos algo para enseñar - el poder para querer hacer-, y no sólo que "nos autoriza" el demostrar capacidad técnica (M. Bonafé; 1999).

Se trata de un arduo proceso colectivo de práctica reflexionada, no exento de una revisión epistemológica y política profunda que permita abordar el conocimiento (materia prima del trabajo de enseñar), no como algo dado, a ser consumido, sino como algo a ser creado y re-creado permanentemente; no como "... un bien cultural trasmitido hereditariamente como un don o socialmente como un capital, sino el resultado de un trabajo del pensamiento (...) a través de la historia, el del niño a través de su aprendizaje..." (Charlot, B., 1986, p. 3).

Recuperar al conocimiento y la cultura en su dimensión de producción histórico-social, de trabajo colectivo transformador, de humanización de los sujetos, constituye un desafío para quienes estamos "autorizados" a guiar el proceso: los docentes.

Y así, probablemente, sentar las bases de nuevas formas de autoridad docente se convierta en una aventura educativa que convoque a los estudiantes volver a sentir y construir lazos de pertenencia a la escuela secundaria. 


\section{Multiplicar las oportunidades de producir conocimientos}

La impronta selectiva de la educación secundaria ha legitimado que sólo algunos - los legítimos destinatarios de su acción educadora - pueden atravesar las innumerables pruebas de rendimiento y egresar con éxito, naturalizando que otros están destinados al fracaso.

Transformar la escuela para algunos en la escuela de y con todos los jóvenes, implica poner en cuestión las categorías ideológicas y políticas a partir de las cuales la escuela secundaria ha construido su proyecto pedagógico. Es decir, reflexionar críticamente sobre el proyecto de enseñanza, la significatividad que tiene para estos estudiantes los conocimientos que ponemos - o no - a su disposición, los sujetos que contribuimos a formar desde los vínculos pedagógicos que establecemos, las normas explícitas e implícitas que constituyen "lo escolar cotidiano", el lugar que la escuela le hace a los aprendizajes y las trayectorias sociales de los jóvenes, etc.

En el complejo desafío de formar adolescentes para el ejercicio pleno de su ciudadanía, la escuela debería acompañar la construcción de la subjetividad adolescente, permitiéndoles disfrutar de experiencias de apropiación y producción de conocimientos que los prepare para el ejercicio efectivo de sus derechos y los enriquezca en sus posibilidades de construir opciones de futuro.

El declamado derecho social a la educación se concreta en una escuela secundaria pensada para que puedan aprender todos los adolescentes y jóvenes. El fracaso, que en la secundaria selectiva era visto como resultado de un demérito individual, cobra un nuevo significado. A la hora de repensar condiciones que hagan posible la universalización de la educación secundaria debemos problematizar el llamado "fracaso escolar", entendiéndolo como falencias de políticas públicas y prácticas escolares quienes "...en su responsabilidad de transmisión de conocimientos y saberes no ha sabido, no ha podido dar las respuestas necesarias y ajustadas a cada uno y una de los/as jóvenes que en ella habitan..." (Fundación Amstrong, 2008, P. 22.)

El fenómeno de la exclusión por la reiteración de experiencias de fracaso afecta fundamentalmente las trayectorias de los estudiantes provenientes de sectores más humildes, que no tienen la opción de acompañar o sustituir su escolaridad por distintos dispositivos de educación privada. Esto nos desafía, en el plano material, a redefinir los modos de selección y organización curricular en función de multiplicar las oportunidades de producir conocimientos como alternativa al "fracaso escolar". Y en la dimensión simbólica, a problematizar nuestras representaciones sobre el conocimiento y la enseñanza a fin de poner a disposición de los estudiantes la posibilidad de construir nuevas estrategias de estudio.

Algunas líneas de acción que podrían iniciar un proceso de transformación tanto de los formatos institucionales, como de la ideología que sustentan ciertas culturas o habitus escolares deberían considerar simultáneamente distintos planos: 
- $\quad$ Asumir que los procesos de enseñanza y los aprendizajes que producen comprometen al conjunto de las instituciones educativas (las escuelas, el sistema y las políticas educativas) y no son sólo de responsabilidad individual (de estudiantes, docentes y/o familias). Por ejemplo, debería reemplazarse el criterio de repitencia por simple acumulación de materias desaprobadas o previas por alguna forma de definición institucional de la promoción.

- Producir rediseños institucionales (en tiempos, espacios, agrupamientos) para permitir el desarrollo de nuevos procesos de enseñanza-aprendizaje. En ese sentido sería deseable que los estudiantes cuenten con la posibilidad de revisar sus aprendizajes en el marco de nuevas experiencias de formación (espacios de acompañamiento o intensificación de estudios) organizadas por la escuela, espacios en los que tendrían la oportunidad de reencontrase con los contenidos no aprendidos a través de nuevas estrategias de enseñanza y nuevas dinámicas de estudio.

- $\quad$ La creación de nuevos puestos de trabajo y asignación de tiempos pagos dentro de la jornada laboral para trabajo institucional a los profesores (para talleres de apoyo, tutorías, seguimiento de trayectorias) deberían definirse en los ámbitos de negociación colectiva correspondientes.

- Ninguna de estas acciones podrá ser llevada a cabo sin que se le ofrezcan a los profesores que asuman responsabilidades en los nuevos espacios curriculares un proceso intensivo de formación docente en servicio. Durante los próximos años esta formación debería iniciarse antes que la implementación de los cambios, e incluso, acompañar el desarrollo de los primeros años a fin de retroalimentar práctica y teoría, para que las transformaciones tengan anclaje en el cotidiano de las escuelas y no sólo en el discurso de las planificaciones.

Al generar tiempos y espacios institucionales de reflexión sobre la propia práctica, se busca que los profesores construyan sus herramientas conceptuales para la redefinición de los modos de organización de los conocimientos y las formas de intervención didácticas. Como lo expresan las siguientes reflexiones respecto del punto de partida y el horizonte que se requiere para producir esta verdadera transformación del marco referencial de la práctica pedagógica de los profesores:

... no resulta hoy visible para los jóvenes - tampoco para muchos adultos- la función del conocimiento como herramienta para pensar la realidad. La distancia cultural que se produce entre los adolescentes y los docentes hace que muchas veces sean descalificadas por la escuela las opciones éticas y estéticas de los estudiantes que se ven obligados a renunciar a una parte importante de sí mismos para ser contemplados en el proyecto educativo (...). 
Estamos convencidos de que la escuela podría ser - lo es sólo en algunos casos y como producto de la voluntad y el compromiso de pequeños colectivos de docentes y directivos- un ámbito en el que los niños y los jóvenes lograran una experiencia sustancial, transformadora, con relación a la percepción que tienen de sus posibilidades intelectuales. Pensamos la escuela como un lugar en el que los jóvenes aprenden a disfrutar de la cultura y de la posibilidad de conocer, a tener la gratificación de sentir que pueden comprender, mirar el mundo con herramientas explicativas y esclarecedoras. Que puedan tomar contacto con los problemas de la sociedad actual y comprender cómo cada campo de conocimientos hace un recorte que ofrece herramientas teóricas que permiten estudiar una parte de esa compleja realidad en la que viven. Para que esto sea posible, consideramos que es necesario concebir al alumno en una posición de producción de conocimiento, lo cual supone-desde nuestro punto de vista, de modo ineludible- un docente que promueve el juego de la producción de conocimientos en la clase, que confía en las posibilidades intelectuales de sus alumnos y que a la vez se piensa a sí mismo como productor... (Sadovsky, P., Espinosa, A. y Bravo, G., 2008, p. 4).

\section{Tiempos y espacios de trabajo colectivo}

Desde una perspectiva antropológica el trabajo refiere a la actividad específica y vital para la autorrealización humana (Marx, 2003). Por ello el trabajador, el productor, es el sujeto creador de los bienes -materiales y simbólicos- que permiten la existencia de la sociedad.

No refiere sólo a un hecho económico -parte del proceso productivo- sino que también forma parte de la trama socio-cultural y política de la sociedad, y por ello incide en las representaciones de los sujetos acerca de sí mismos. La práctica social de los individuos y grupos combina el mundo de la educación y el del trabajo de modo simultáneo, y es desde este reconocimiento que se plantea la necesidad de considerarlo como un constructo social-político.

El trabajo es cooperativo, social, no tiene otra forma de realización que mediante la unión de los individuos. Sin embargo, en la sociedad capitalista, el trabajo puede convertirse y de hecho se transforma generalmente en un elemento alienante y opresivo.

Los modos tradicionales de organización de la "tarea docente" en el nivel secundario, funcionales a la organización capitalista del trabajo asalariado, invisibilizan la dimensión de trabajo que tiene el hecho educativo y las prácticas pedagógicas. En particular, queda oculto el carácter colectivo del trabajo docente.

A esta fragmentación le resulta funcional una organización del trabajo sustentada en un currículo por "materias" o "asignaturas", centrado en el trabajo áulico, con jornadas laborales medidas en "horas clase", un salario que se compone por la acumulación de esas horas que implican la atención de cientos de 
alumnos por semana (que en ese contexto sólo logran aparecer como apellidos en una lista). Trabajo docente que sólo es retribuido en su carácter de "frente a alumnos", y excepcionalmente sólo la planificación, la preparación de clases y la corrección de evaluaciones son consideradas otras tareas inherentes a la función de enseñar. De este modo quedan por fuera del reconocimiento como parte del proceso de trabajo, y por tanto no se pagan, actividades tales como formación en servicio, proyectos pedagógicos e institucionales, reuniones departamentales o de intercambio entre docentes, etc; no casualmente aquellas que apuntan a fortalecer procesos de articulación de los conocimientos, de los grupos de estudiantes y de profesores, de la escuela con su contexto.

La falta de resolución de esta cuestión y la imposibilidad de materializar tiempos y espacios de trabajo docente colectivo ha obstaculizado la mayoría de las estrategias de transformación educativa y fundamentalmente aquellas que buscan profundizar los cambios en la educación secundaria de manera democrática.

La reforma neoliberal de los noventa profundizó la fragmentación, el verticalismo, el carácter instrumental del trabajo docente y a la vez el estilo tecnocrático de implementación de las reformas, donde el "conocimiento escolar" era el legitimado por los expertos. En un mundo complejo, se complejizaba la tarea del educador, a la par que se precarizaban sus condiciones laborales y salariales, cuyas consecuencias fueron el deterioro del ánimo y la salud, la (auto) desvalorización sobre su propio trabajo y su imagen social.

En este contexto se explica fácilmente la naturalización entre los docentes de las escuelas secundarias de:

- una formación docente que minimiza los "saberes del enseñar" en nombre de la superioridad de los "contenidos";

- $\quad$ una estructura curricular fundada en la fragmentación del abordaje de la realidad a través de las "asignaturas" (versión degradada de los campos disciplinarios);

- $\quad$ un discurso jerarquizante sobre la autoridad académica, cuyos escalones se ordenan según los niveles del sistema.

A partir de estas posiciones consideramos que develar la naturaleza colectiva del trabajo docente significa una marca re-fundacional de la escuela secundaria en tanto confronta con las concepciones que naturalizan la apropiación privada de todo lo que ese trabajo produce, en particular de la cultura y el conocimiento. Y a la vez pone en cuestión la matriz organizacional tradicional de la escuela secundaria: currículo clasificado (por asignaturas) + formación docente especializada (por disciplinas) + contrato de trabajo de los profesores por horas cátedra.

Afirmar dicho carácter colectivo requiere pensar en conjunto el trabajo de los profesores, la organización de las escuelas, y el currículo que cada institución despliega. Es decir, que estén garantizadas las condiciones materiales 
(espacios, tiempos, recursos) para que profesores, auxiliares docentes y estudiantes puedan ser sujetos de procesos de trabajo que, aunque diferenciados y específicos, confluyan en la concreción de un proyecto educativo común.

\section{Breves reflexiones finales}

Como cierre de este artículo me gustaría recuperar la idea de que estamos transitando tiempos de crisis y desafíos. Si bien es imposible dimensionar hoy qué tanto hay de "lo viejo que no termina de morir y lo nuevo que no termina de nacer", no dejan de resultar tiempos apasionantes, de debates fuertes y de posibilidades abiertas.

Sería importante que en esta construcción se logre involucrar al conjunto de la ciudadanía. En una democracia, las políticas educativas no pueden ser sólo patrimonio de un gobierno, ni de un staff de funcionarios técnicos y mucho menos de las corporaciones que hacen lobby con el único fin de satisfacer sus rentabilidades. Como lo han demostrado leyes recientes cuya sanción e implementación implican cambios culturales, su fortaleza reside en la capacidad de movilizar a la sociedad en un sentido transformador.

Contravenir el modelo pedagógico de una educación secundaria que nació selectiva, vertical y meritocrática, y que se fue precarizando hasta convertirse en una mala copia de sí misma, es una de esas batallas culturales. Batalla que nos involucra como trabajadores de la educación y nos desafía a transformar la vieja educación media en una aventura educativa que convoque a los estudiantes re-fundar su pertenencia a esta, otra, escuela secundaria necesaria y posible.

\section{Notas Bibliográficas}

(1) "Experiencias pedagógicas populares en la escuela secundaria. Sistematización y análisis de cuatro casos “ (2008-2011). Proyecto de Investigación que tiene como objeto la sistematización y el análisis de las experiencias educativas desarrolladas en cuatro escuelas secundarias, situadas en Río Negro, Chaco, Buenos Aires y Capital Federal. Dirección: Silvia Andrea Vázquez. Codirección: Susana Di Pietro. Investigadoras: Lila Ana Ferro, Andrea Nuñez, María Dolores Abal Medina y Mariano Indart.

(2) En 1914 el porcentaje de la población de 13 a 18 años matriculado en la enseñanza media era del $3 \%$. En tanto, el 48 \% de los niños de 6 a 14 años se encontraban matriculados en la escuela. (Tedesco, Juan Carlos, 1980: 113-114).

(3) Censo de Población 1960. Citado en: Paviglianitti, Norma (1988).

(4) La tasa de crecimiento global en cada subperíodo es prácticamente la misma: de un $39,42 \%$ entre 1960 y 1980; y del 39,66 \% entre este último año y el 2001.

(5) La tasa de crecimiento es sensiblemente mayor para el nivel secundario que para el crecimiento total. Y también es mayor para el primer subperíodo (del $70 \%$ ) que para el segundo, donde fue de $60 \%$. 


\section{Referencias Bibliográficas}

- Balduzzi, J. y otros. (2006). Desigualdad y exclusión en la educación secundaria de los adolescentes y jóvenes. Informes y estudios sobre la situación educativa $N^{\circ} 4$. Buenos Aires: IIPMV- CTERA.

- $\quad$ Charlot, B. (2008). La relación con el saber. Elementos para una teoría. Buenos Aires: Libros del Zorzal.

- Charlot, B (1986). La epistemología implícita en las prácticas de enseñanza de las matemáticas._Conferencia dictada en Cannes. Mimeo

- $\quad$ CTERA. (2008). Otra escuela secundaria es necesaria y posible. Buenos Aires.

- $\quad$ Fundación Amstrong. (2008. Proyecto Escuela Secundaria La Salle. Dipregep 6557. Pcia. de Bs As. Mimeo.

- González, H. y otros. (2009). Reconociendo nuestro trabajo docente. Bs. As.: Ediciones CTERA.

- Jacinto, C. y Terigi, F. (2007). ¿Qué hacer ante las desigualdades en la educación secundaria?. Aportes de la experiencia latinoamericana. Buenos Aires: Santillana.

- Martínez Bonafé, J. (1999). Trabajar en la escuela. Bs. As: Miño y Dávila.

- $\quad$ Marx, K. (2003). El Capital. Tomo I. Siglo XXI. Buenos Aires.

- Sadovsky, P., Espinosa, A. y Bravo, G. (2008). Una propuesta de Investigación para la Escuela Secundaria. Reseña, mimeo. SUTEBA.

- de Sousa Santos, B. (2003). Crítica de la razón indolente: contra el desperdicio de la experiencia. España: Desclee de Brouwer.

- Santore, M. (2010). La autoridad pedagógica de los docentes en tiempos de adolescencia cultural. CTERA. mimeo.

- $\quad$ Santore, M., Maldonado, S. Ojea M., y Vázquez, S. A. (2011). Autorizados a enseñar. Diálogos y relatos sobre la autoridad de los docentes. Bs. As: Ediciones CTERA.

- $\quad$ Spinosa, M. (2006). Los saberes y el trabajo. Ensayo sobre una articulación posible. Revista Anales de la Educación Común. 2 (3) (pp. 164 a 173). DGCyE. Buenos Aires.

- Tiramonti, G. y Montes, N. (comp.). (2009.) La escuela media en debate. Problemas actuales y perspectivas desde la investigación. Buenos Aires / FLACSO, Manantial.

- Torres Santomé, J. (1994.) El currículo integrado. Madrid, Morata.

- Vázquez, S. A. (2009) Nadie aprende repitiendo. Multiplicar las oportunidades y las experiencias de aprendizaje. Informes y estudios sobre la situación educativa №9 -IIPMV-CTERA.

- Vázquez, S. A. (2010). La transformación de la educación secundaria. De los enunciados políticos a las prácticas. Revista Voces en el Fénix. 1 (3). Disponible en http://www. vocesenelfenix.com/pauta2209finalweb.pdf 